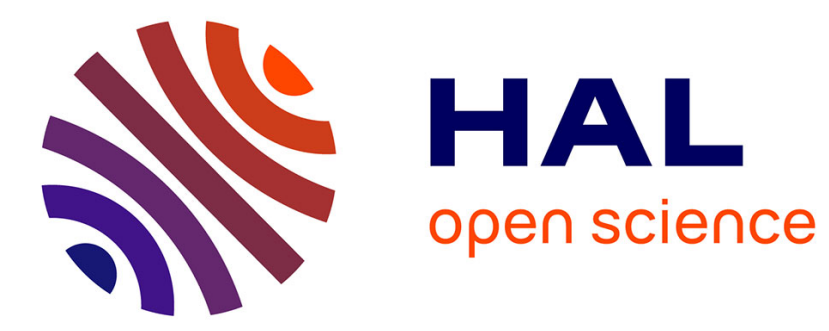

\title{
Fiber tracking on HARDI data using robust ODF fields
} Haz-Edine Assemlal, David Tschumperlé, Luc Brun

\section{To cite this version:}

Haz-Edine Assemlal, David Tschumperlé, Luc Brun. Fiber tracking on HARDI data using robust ODF fields. IEEE International Conference on Image Processing, Sep 2007, San Antonio, United States. pp.133-136, 10.1109/ICIP.2007.4379264 . hal-00250226

\section{HAL Id: hal-00250226 \\ https://hal.science/hal-00250226}

Submitted on 11 Feb 2008

HAL is a multi-disciplinary open access archive for the deposit and dissemination of scientific research documents, whether they are published or not. The documents may come from teaching and research institutions in France or abroad, or from public or private research centers.
L'archive ouverte pluridisciplinaire $\mathbf{H A L}$, est destinée au dépôt et à la diffusion de documents scientifiques de niveau recherche, publiés ou non, émanant des établissements d'enseignement et de recherche français ou étrangers, des laboratoires publics ou privés. 


\title{
FIBER TRACKING ON HARDI DATA USING ROBUST ODF FIELDS
}

\author{
H-E. Assemlal \\ D. Tschumperlé \\ L. Brun

\section{GREYC IMAGE (CNRS UMR 6072), 6 Bd Maréchal Juin, 14050 Caen Cedex, France}

\begin{abstract}
We present a robust method to retrieve neuronal fibers in human brain white matter from High-Angular Resolution MRI (HARDI datasets). Contrary to classical fiber-tracking techniques done on the traditional 2nd-order tensor model (DTI) which may lead to truncated or biased estimated diffusion directions in case of fiber crossing configurations, we propose here a more complex approach based on a variational estimation of Orientation Diffusion Functions (ODF) modeled with spherical harmonics. This kind of model can correctly retrieve multiple fiber directions corresponding to underlying intra-voxel fibers populations. Our technique is able to consider the Rician noise model of the MRI acquisition in order to better estimate the white matter fiber tracks. Results on both synthetic and real human brain white matter HARDI datasets illustrate the effectiveness of the proposed approach.
\end{abstract}

Index Terms - Diffusion MRI, Variational methods and PDEs, ODF estimation, Fiber-tracking

\section{INTRODUCTION}

Diffusion Magnetic Resonance Imaging (dMRI) [1] is a noninvasive method to observe the Brownian motion of water molecules constrained by neuronal tissues in vivo within the brain. Diffusion Tensor Imaging (DTI) is a well-known particular case of such a modality which maps each voxel signal to a 2nd-order tensor model [2]. It implicitly assumes that the diffusion is Gaussian everywhere, which is wrong and leads to serious limitations when estimating intra-voxel diffusion configurations where more than one single fiber direction predominates, like in crossing or kissing fibers patterns. In order to overtake this significant shortcoming, higher order diffusion model have been considered so far. Historically, Stejskal and Tanner were the first to show the exact relation between the diffusion signal and the diffusion probability density function (PDF) [3]. More recently, Tuch proposed the Q-Space Imaging (QSI) technique based on the inverse Fourier Transform to estimate the PDF. Unfortunately, this method has significant restrictions essentially because of the long acquisition time needed to sample the whole q-space. Considering QSI limitations, High Angular Resolution Diffusion Imaging (HARDI) comes as an interesting alternative as it samples the diffusion signal only on the single sphere following discrete gradient directions; and consequently needs less time. Liu et al. [4] proposed a generalization of DTI based on the expansion of Fick's diffusion laws to higher order. However in practice, this methods requires to sample several q-space single spheres at different gradient strenghts and undesirably increases the number of acquisitions. Tuch in [5] proposed to measure diffusion orientation through the Orientation Distribution Function (ODF) defined as the radial projection of the spherical diffusion function from HARDI data. Given a unit spatial direction $\mathbf{u} \in \mathbb{R}^{3}, \Psi(\mathbf{u})$ is the radial projection of the PDF on the line directed by $\mathbf{u}$. Thus, the exact ODF $\Psi$ can be written without loss of generality with $\mathbf{u}$ taken as the $\mathrm{z}$-axis, as

$$
\Psi(\mathbf{u})=\int_{o}^{\infty} P(\alpha \mathbf{u}) d \alpha=\int P(r, \theta, z) \delta(\theta, z) r d r d \theta d z
$$

Tuch [5] showed that the Funk-Radon transform (FRT) $\mathcal{G}$ from the raw HARDI data approximates the ODF on the Q-space single sphere:

$$
\mathcal{G}_{q^{\prime}}[S(\mathbf{q})](\mathbf{u})=2 \pi q^{\prime} \int P(r, \theta, z) J_{0}\left(2 \pi q^{\prime} r\right) r d r d \theta d z
$$

where $J_{0}$ stands for the zeroth-order Bessel function. Consequently, the estimated ODF in a direction $\mathbf{u}$ is given by the great integral over the diffusion signal on the plane orthogonal to $\mathbf{u}$. This leads to an interesting model-free method known as Q-Ball Imaging to retrieve orientation diffusion informations, contrary to the model-based methods which implies a strong a priori knowledge about the local fiber configuration. Once having estimated diffusion directions, an interesting application of diffusion MRI consists in retrieving neuronal fibers in brain white matter by the mean of a so called fiber-tracking algorithm. This is classically done by computing the integral curve of interpolated DTI dominant eigenvectors [6, 7]. However, these methods are very sensitive to noise since it always suppose that the dominant eigenvector is correct. Noise issue was tackled in [7, 8, 9] who proposed to apply regularization schemes on tensor or principal direction before applying the fiber-tracking step. One of the main limitation of the DTI model is that it is not able to retrieve several intra-voxel fiber distributions, leading to wrong or biased estimation of dominant fiber directions. On the other hand, recent higher order models as ODF fields are promising for estimating correct neuronal fibers.

In the following sections, we quickly remind the linear estimation technique of the ODFs introduced by Descoteaux et al. [10] (section 2.1). In section 2.2, we present our contribution, i.e. a new variational framework for a more robust 
estimation of the ODF field. It has the advantage of being nonlinear, allowing to estimate and regularize simultaneously a whole volume of ODFs. We highlight the importance of a robust ODF estimation considering regularization constraints on fiber-tracking in section 3 . We finally illustrate this model by validating results on synthetic and human brain HARDI data.

\section{ROBUST ESTIMATION}

\subsection{Linear estimation}

Descoteaux et al. [10] recently proposed an elegant analytical method based on the Funk-Hecke theorem to calculate the great integral of the FRT from a signal expressed in a spherical harmonics $(\mathrm{SH})$ modified basis. It is a set of orthonormal functions to describe complex functions defined on the unit sphere and constrained to be symmetric and real [10, 11, 12] as these are known diffusion signal properties. Thus, let $Y_{j}$ of degree $j$ be a spherical harmonic, any function $\chi$ defined on the unit sphere $\forall(\theta, \phi) \in \Omega_{\chi}=[0, \pi] \times[0,2 \pi), \chi: \Omega_{\chi} \rightarrow \mathbb{R}$ can be described as:

$$
\chi(\theta, \phi)=\sum_{j=0}^{N} c_{j} Y_{j}(\theta, \phi)=\tilde{B} C_{j_{(\mathbf{p})}}\left(\theta_{i}, \phi_{i}\right)
$$

where $N$ corresponds to the highest degree of the decomposition into spherical harmonics, $\tilde{B}$ is a matrix of $\mathrm{SH}$ functions $Y_{j}$ and $\mathbf{C}: \mathbb{R}^{3} \rightarrow \mathbb{R}^{N}$ be the vector of coefficients of spherical harmonics at voxel $\mathbf{p}=(x, y, z)$.

Let $\mathbf{S}: \mathbb{R}^{3} \rightarrow \mathbb{R}^{n_{s}}$ be the vector field of diffusion signal in $n_{s}$ discrete directions on the sphere. Descoteaux et al. [10] proposed to fit the signal with a continuous spherical function by a least square minimization

$$
\min _{\mathbf{C} \in \Omega_{S}}\left\|\mathbf{S}_{(\mathbf{p})}\left(\theta_{i}, \phi_{i}\right)-\tilde{B} \mathbf{C}_{(\mathbf{p})}\left(\theta_{i}, \phi_{i}\right)\right\|^{2}
$$

where $\theta_{i}, \phi_{i}$ follow gradient discretization of the diffusion signal on the single sphere. Best fitting coefficients $\mathbf{C}$ are then given by a modified Moore-Penrose pseudo-inverse scheme.

$$
\mathbf{C}_{(\mathbf{p})}=\left(\tilde{B}^{T} \tilde{B}+\lambda \tilde{L}\right)^{-1} \tilde{B}^{T} \mathbf{S}_{(\mathbf{p})}
$$

where $\lambda$ is the weight term on the frequential regularization matrix $\tilde{L}$. At this point, we have a continuous spherical function fitting the diffusion signal. We want now to recover the ODF which gives the orientation of the diffusion. Descoteaux et al. [10] showed that the FRT approximating the ODF can be expressed using the $\mathrm{SH}$ basis, by:

$$
\mathcal{G}_{q^{\prime}}\left[\mathbf{S}_{(\mathbf{p})}(\mathbf{q})\right]=\tilde{P} \tilde{B} \mathbf{C}_{(\mathbf{p})}=\sum_{j}\left[2 \pi \frac{P_{l_{j}}(0)}{P_{l_{j}}(1)}\right] c_{j_{(\mathbf{p})}} Y_{j_{(\mathbf{p})}}
$$

where $\tilde{P}$ a $N$-rank order diagonal matrix, and $P_{l_{j}}$ are associated Legendre polynomials at order $l_{j}$ (value of $l$ knowing $j$ ). $\tilde{P}$ is a transition matrix from Q-space signal to diffusion probability space.

The spherical harmonics are a powerful tool to recover an approximation of the ODF. However, MRI noise distribution follows a Rice distribution [13] not a Gaussian one. Therefore, a least square fit is definitely not the best choice for such an estimation process. Furthermore, estimation is made voxel-by-voxel and does not reflect the spatial regularity of the diffusion function. Hence, our contribution is a variational framework which is adaptable to MRI noise distribution and able to use valuable informations of the neighbour voxels.

\subsection{PDE-based estimation}

The key idea is to estimate and regularize the whole volume of voxels at the same time. It is worth to mention that similar methods have been proposed for the regularization of DTI [9. 14] and apparent diffusion coefficient (ADC) [15]; yet none is able to take advantage of the informations provided by the ODFs.

Let $\mathbf{C}: \Omega_{C} \subset \mathbb{R}^{3} \rightarrow \mathbb{R}^{N}$ be the volume of spherical harmonics coefficients, $n_{s} \in \mathbb{R}$ be the number of gradient directions and $\tilde{B}$ be the matrix of size $\left(n_{s}, N\right)$

$$
\tilde{B}=\left[\begin{array}{ccc}
Y_{1}\left(\theta_{1}, \phi_{1}\right) & \ldots & Y_{N}\left(\theta_{1}, \phi_{1}\right) \\
\vdots & \ddots & \vdots \\
Y_{1}\left(\theta_{n_{s}}, \phi_{n_{s}}\right) & \ldots & Y_{N}\left(\theta_{n_{s}}, \phi_{n_{s}}\right)
\end{array}\right]
$$

We propose to robustly estimate and regularize the ODF field simultaneously by minimizing this nonlinear functional energy $E$ defined as:

$$
\min _{\mathbf{C} \in \Omega_{C}}\left\{E(\mathbf{C})=\int_{\Omega_{S}}\left[\sum_{k}^{n_{s}} \psi\left(\left|\mathbf{D}_{k}\right|\right)\right]+\alpha \varphi(\|\nabla \mathbf{C}\|) d \Omega_{S}\right\}
$$

where $\mathbf{D}_{k}$ at voxel $\mathbf{p}$ is $\mathbf{D}_{k_{(\mathbf{p})}}=\mathbf{S}_{k_{(\mathbf{p})}}-\sum_{j} \tilde{P}_{j}^{-1} \tilde{B}_{k, j} \mathbf{C}_{j_{(\mathbf{p})}}$ is a data attachment term which measures the differences between the raw signal and its ODF estimation at gradient direction $k, \psi: \mathbb{R} \rightarrow \mathbb{R}^{+}$and $\varphi: \mathbb{R} \rightarrow \mathbb{R}^{+}$are real and positive functions, $\alpha \in \mathbb{R}$ is the regularization weight and $\|\nabla \mathbf{C}\|$ the gradient norm defined as $\|\nabla \mathbf{C}\|=\sum_{j}\left\|\nabla C_{j}\right\|$. Note that if $\psi(s)=s^{2}$ and $\alpha=0$ in $(8)$, we minimize the LS criterion (5, corresponding to the Descoteaux's method with $\lambda=0$ ). Yet, as MRI noise follows a Rician distribution, least square criterion is not the best choice. The $\psi$ function is defined to support a robust ODF estimation and regularization preserves contours between different fiber distribution regions using the gradient norm $\nabla\|\mathbf{C}\|$. Indeed, Frank in [12] points out that the spherical harmonics basis is well adapted to characterize anisotropy since its coefficients characterize isotropic $(j=0)$, one-fiber $(j=1)$, and several fibers $(j>=2)$ diffusions. As the minimization cannot be computed straightforwardly, the gradient descent coming from the Euler-Lagrange derivation of (8) leads to a set of multi-valued partial derivate equation (PDE) (9). In order to estimate a solution, $\mathrm{SH}$ coefficients velocity $\frac{\partial \boldsymbol{C}}{\partial t}$ giving the direction from the current $\mathbf{C}_{t}$ to a solution is computed. The latter is done several times until 
convergence (typically when $\varepsilon \rightarrow 0, \frac{\partial \mathbf{C}}{\partial t}<\varepsilon$,).

$$
\begin{aligned}
\frac{\partial \mathbf{C}_{j}}{\partial t} & =\tilde{P}_{j}^{-1} \sum_{k} \psi^{\prime}\left(\left|\mathbf{D}_{k}\right|\right) \operatorname{sign}\left(\mathbf{D}_{k}\right) \tilde{B}_{k, j} \\
& +\alpha \operatorname{div}(\varphi(\| \nabla \mathbf{C}||))
\end{aligned}
$$

The initial estimate $\mathbf{C}_{t=0}=\mathbf{U}_{0}$ is computed either by considering a random field or a more structured one. A good choice is to start from an initial set which is not so far from the global minimum; so the linear LS estimation (5) seems to be an adequate alternative. Indeed, LS minimization is the global minimum when $\psi(s)=s^{2}$ and $\alpha=0$. One can expect the minimum to be close enough to the LS minimum through variations of $\psi$ and $\varphi$ (c.f. Fig.1.(e/f)); and should consequently bring down the number of iterations required to converge.

\section{FIBER-TRACKING}

DTI-based fiber-tracking has been widely used [6, 7, 8, 9 but it has significant drawbacks when dealing with intra-voxel structures. Indeed, not only DTI cannot model crossing or kissing fibers but it also estimates wrong directions in the case of multiple fiber configurations. On the contrary, ODF does not fall into this restrictions. Nevertheless, although the issue of robust fiber-tracking has received numerous contribution with DTI model it is still an open problem when using ODFs. In order to illustrate the influence of a robust ODF estimation on fiber-tracking, we propose a model for retrieving neuronal fiber in brain white matter.

A way to do fiber-tracking is to use estimated displacement due to diffusion which is given by the ODF in order to find dominant directions. Once directions are retrieved, only one is kept based on a a priori on the fibers distribution, resulting in a diffusion tensors field $\mathbf{w}$. A line integration scheme is needed to propagate a fiber along a curve $\mathcal{C}$ through the tensors volume (c.f. Fig.11). One may want to use Euler method

$$
\mathcal{C}_{a+h}=\mathcal{C}_{a}+h \mathbf{w}_{a}+O\left(h^{2}\right)
$$

where $a$ is the current position in the curve $\mathcal{C}$ and $h$ is the integration step. In practice, Euler's method is not stable and precise and so Runge-Kutta comes as an interesting alternative. This method can be seen as the result of reduction in precision of a curve $\mathcal{C}^{\prime}$ more precise than $\mathcal{C}$ because of a smaller integration step

$$
\mathcal{C}_{a+h}=\mathcal{C}_{a}+\frac{k_{1}}{6}+\frac{k_{2}}{3}+\frac{k_{3}}{3}+\frac{k_{4}}{6}+O\left(h^{5}\right)
$$

where $k_{i}$ are the slope estimated in $a+i / 4 h$. Actually, fourthorder Runge-Kutta is by far the most precise and is the one we used on our tests.

Besides, we assume that there are no neuronal fibers in water regions of the brain, and consequently there is a need to identify this regions. Generalized Fractional Anisotropy (GFA) (c.f. bottom of Fig.1. a) as proposed by Tuch in [5] measures the variation within the diffusion as a spherical function. It can be expressed in the spherical harmonics basis which has the advantage to be much faster to compute.

$$
G F A=\frac{s t d(\Psi)}{r m s(\Psi)}=\sqrt{1-\frac{c_{0}^{2}}{\sum_{j=0}^{N} c_{j}^{2}}}
$$

This gives a convenient way to measure apart isotropic from anisotropic area; therefore we used it to stop fiber line integration when arriving in water area, i.e. when $G F A$ is below a threshold.

\section{APPLICATIONS}

For all our experiments, we used the robust estimation function $\psi(s)=1-e^{\left(-\frac{s^{2}}{\kappa_{1}}\right)}$ and the discontinuity-preserving regularization function $\varphi(s)=\frac{1}{1+s^{2} / \kappa_{2}}$, where $\kappa_{1}$ and $\kappa_{2}$ are two thresholds depending on the value range of the original HARDI dataset. Please refer to [16] for a function $\psi$ specific to MRI Rician noise. We first present results of our variational framework on synthetical HARDI data created using a Gaussian multi tensor model [11] to simulate $n$ fibers crossing. Discretization of the sphere (72 directions) was obtained from the subdivision of a regular icosahedron. Our synthetic data simulate horizontal and vertical fibers (respectively right and top in Fig.11) merging into one horizontal fiber (left in Fig.11). From the several fibers distributions estimated, we retrieved one using a simple a priori, i.e. to follow the direction which is the most vertical. As expected, DTI is not able to retrieve correctly the profile of any underlying fiber as shown in Fig.(1.b). Instead, it estimates a wrong direction, which is a mixture of the two main directions from each fiber distribution. Therefore the estimated fiber is a fictive one since a correct path in this dataset would be either horizontal or going vertical. Fiber-tracking on ODF does not have this problem, but it is sensitive to noise. However our variational method successfully estimates the ODFs field from noisy data $(P S N R=15 d B)$, which leads to good fiber-tracking (c.f. Fig.1.(e/f)).

We finally tested our estimation framework on a human brain HARDI dataset, using a $1.5 T$ MRI scanner with 31 gradient directions and $b=500 \mathrm{~s} / \mathrm{mm}^{2}$. A comparison between DTI, linear estimation and our variational framework is shown in Fig. 2 on an interesting brain white matter region as it is the meeting place of several fibers. Our regularized three dimensional estimation performs an enhancement of the contrast of the diffusion function when there are underlying fibers, and keeps water regions isotropic.

\section{CONCLUSION}

We proposed a robust tractography method with the use of variational scheme to estimate ODFs from HARDI data. This greatly improves the performance and the precision of the results on very preliminary MRI noisy data. The ability to recover reliable and accurate intra-voxel fibers distributions within the human brain is promising and opens new perspectives for studying more precisely the neuronal fiber network. 


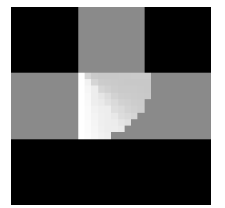

(a) GFA

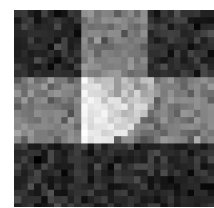

(d) noisy GFA

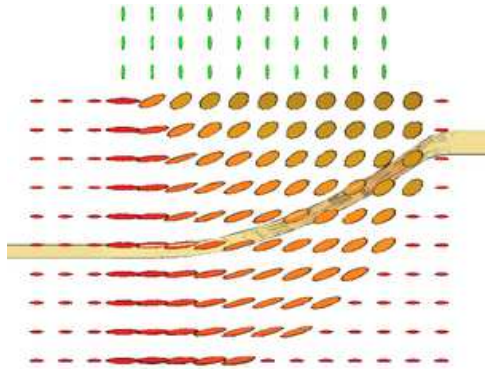

(b) DTI

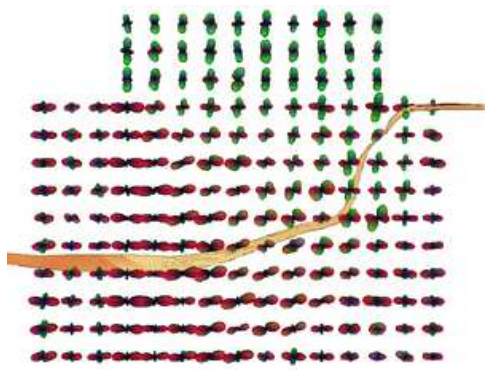

(e) noisy LS

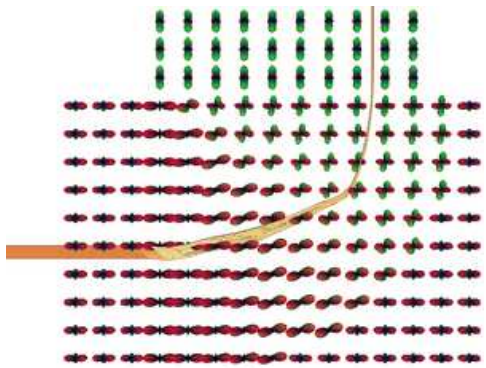

(c) LS

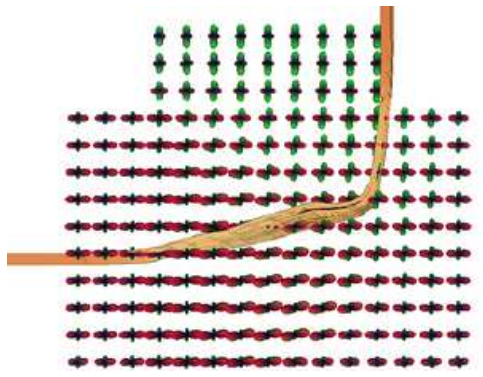

(f) noisy PDE

Fig. 1. Crossing fibers distributions: estimation and fiber-tracking.

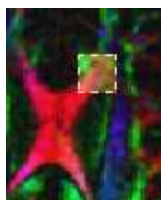

(a) FA

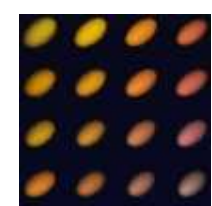

(b) DTI

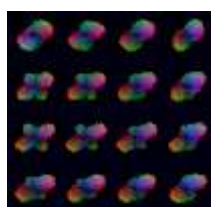

(c) LS

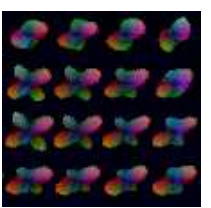

(d) PDE

Fig. 2. Comparison of estimation on frontal genu corpus callosum meeting frontal gyrus.

\section{Acknowledgments}

The authors thanks CHU of Caen and GIN Cyceron for their data and the fruitful discussions.

\section{REFERENCES}

[1] D. LeBihan, E. Breton, D. Lallemand, et al., "Mri of intravoxel incoherent motions: Application to diffusion and perfusion in neurologic disorders," Radiology, pp. 401-407, 1986.

[2] P.J. Basser, J. Mattiello, and D. LeBihan, "Mr diffusion tensor spectroscopy and imaging," Biophysical Journal, vol. 66, no. 1, pp. 256-267, 1994.

[3] E.O. Stejskal and J.E. Tanner, "Spin diffusion measurements: spin echoes in the presence of a time-dependent field gradient," Journal of Chemical Physics, vol. 42, no. 1, pp. 288-292, 1965.

[4] C. Liu, R. Bammer, B. Acar, and M.E. Moseley, "Characterizing non-gaussian diffusion by using generalized diffusion tensors," Magn. Res. in Med., vol. 51, pp. 924-937, 2004.

[5] D.S. Tuch, "Q-ball imaging," Magn. Res. in Med., vol. 52, no. 6, pp. 1358-1372, 2004.

[6] T.E. Conturo, N.F. Lori, T.S. Cull, et al., "Tracking neuronal fiber pathways in the living human brain," NAS of the USA, 1999, pp. 10422-10427.

[7] B.C. Vemuri, Y. Chen, M. Rao, et al., "Fiber tract mapping from diffusion tensor mri," Vancouver, Canada, 2001, VLSM.

[8] O. Coulon, D.C. Alexander, and S.R. Arridge, "A regularization scheme for diffusion tensor magnetic resonance images," Davis,USA, 2001, ICIP, pp. 92-105.

[9] D. Tschumperle and R. Deriche, "Variational frameworks for dt-mri estimation, regularization and visualization," Nice, France, 2003, ICCV.

[10] M. Descoteaux, E. Angelino, S. Fitzgibbons, and R. Deriche, "Regularized, Fast and Robust Analytical Q-Ball Imaging," Magn. Res. in Med., vol. 58, pp. 497-510, 2007.

[11] D.C. Alexander, G.J. Barker, and S.R. Arridge, "Detection and modeling of non-gaussian adc profiles in human brain data," Magn. Res. in Med., vol. 48, no. 2, pp. 331-340, 2002.

[12] L.R. Frank, "Characterization of anisotropy in high angular resolution diffusion-weighted mri," Magn. Res. Med, vol. 47, pp. 1083-1099, 2002.

[13] H. Gudbjartsson and S. Patz, "The rician distribution of noisy mri data.," Magn. Reson. Med., vol. 34, pp. 910-914, 1995.

[14] A. Ramirez-Manzanares and M. Rivera, "A method for estimating brain nerve bundles, by restoring and filtering intravoxel information in dt mri data," Nice, France, 2003, VLSM.

[15] Y. Chen, W. Guo, Q. Zeng, et al., "Estimation, smoothing, and characterization of adc profiles from hardi," CVPR, vol. 1, pp. 588-593, 2004.

[16] H-E. Assemlal, D. Tschumperlé, L. Brun, "A Variational Framework for the Robust Estimation of ODFs From HARDI", Technical report, Les cahiers du GREYC, 2007. 